\title{
WCAG Formalization with W3C Techniques
}

\author{
Vicente Luque Centeno ${ }^{1}$, Carlos Delgado Kloos ${ }^{1}$, \\ Martin Gaedke ${ }^{2}$, and Martin Nussbaumer ${ }^{2}$ \\ 1 Carlos III University of Madrid \\ $\{$ vlc, cdk\}@it.uc3m.es \\ 2 University of Karlsruhe \\ \{gaedke, nussbaumer\}@tm.uni-karlsruhe.de
}

\begin{abstract}
Web accessibility consists of a set of restrictions that Web pages should follow in order to be functional for different devices and users. These restrictions, which are quite heterogeneous and rather expensive to evaluate, unless relayed to human judgement, are usually expressed within a program's code. Different solutions have recently emerged to express these restrictions in a more declarative way. We present a comparison of some of them and propose some W3C techniques for expressing these constraints. Using W3C technologies, the evaluation cost can be clearly minimized.
\end{abstract}

\section{Accessibility}

WAI (Web Accessibility Initiative)'s WCAG (Web Content Accessibility Guidelines) $1.0[1,2]$ is an important contribution to Web accessibility, focusing not only on eliminating barriers for disabled people, but also a major step towards device independence, allowing Web interoperability to be independent from devices, browsers or operating systems. WCAG 1.0 have become an important reference for Web accessibility in the Web community. However the set of the 65 WCAG's checkpoints that accessible documents have to pass is a very heterogeneous set of conditions whose evaluation and repair is difficult to evaluate. WCAG 1.0 specification is written in a high abstraction level which is frequently quite far away from the low level technical detail of the HTML format. Many of those checkpoints are also open to subjective interpretation, including implicit conditions or, simply, containing conditions whose detection can not be automated.

\section{WCAG Evaluability}

Several rules can not be evaluated automatically by a program with an acceptable degree of trust because they require human judgement. However, there are some automatable rules that can be automatically evaluated with a computer's program. Typical evaluation tools $[8,9,10]$ automate the evaluation of these restrictions with their own program's code. XML-based APIs like DOM [5], 
SAX [6] or JDOM [7] can be used in programming languages like Java or Haskell as long as Web pages get a similar aspect as a XML file. However, this involves several difficulties and differences on how their programmers interpret this set of rules. As a result, tools really implement their own set of constraints which are based on the original from W3C, but having important differences from each other that result on different evaluation results, even for the same document, depending on which tool is being used.

The need for a declarative set of restrictions for expressing constraints within Web documents has been recently addressed by some previous work. Taw [8] has had an attempt to express this rule in an internal XML file based on regular expressions that should match document's markup, but the expresivity power was really poor and regular expressions maintainability was not very cost effective. Kwaresmi [11] recently used a self-developed XML-based language for expressing accessibility constraints. It was based on the document's structure (not the document's markup itself), so things like the order of the attributes within a tag, uppercase or lowercase sensitivity or white-spaces became properly treated, among others. However, this XML-based language results rather verbose and is no more than a $a d$-hoc developed format specifically devoted to express accessibility constraints. More generic approaches can be achieved with generic XML-based restriction languages like Xlinkit [12] which is a first-order logic XML-based language for generic restrictions on XML documents. This language could effectively be used to express declarative rules that are automatically checked by a generic validation tool that spots all XML nodes that do not follow the declared restrictions. Each failing node is spotted with a unique XPath locator that facilitates where accessibility barriers may be found. Xlinkit provides evaluating constructors for first-order logic operators including conditional, comparison and quantified expressions. Though this solution is generic and powerful, tools incorporating Xlinkit should use their implementor's platform.

In front of these related works, we have found that XPath 1.0 [3] and XQuery 1.0 [4] may be used to express accessibility restrictions. Table 1 shows several XPath locators for nodes breaking some WCAG checkpoints. Other XQuery 1.0 based expressions can be found at [13].

Table 1. XPath 1.0 locators for nodes breaking accessibility constrains

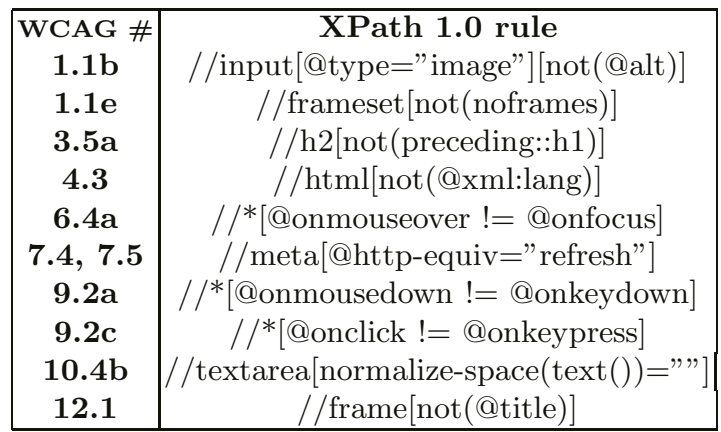




\section{Acknowledgements}

The work reported in this paper has been partially funded by the projects INFOFLEX TIC2003-07208 and SIEMPRE TIC2002-03635 of the Spanish Ministry of Science and Research.

\section{References}

1. W3C Web Content Accessibility Guidelines 1.0 www . w3. org/TR/WCAG10

2. W3C Techniques For Accessibility Evaluation And Repair Tools W3C Working Draft, 26 April 2000

www. w3. org/TR/AERT

3. W3C XML Path Language (XPath) Version 1.0 W3C Recommendation 16 November 1999

www.w3.org/TR/xpath

4. W3C XQuery 1.0: An XML Query Language W3C Working Draft 29 October 2004 www.w3.org/TR/xquery

5. W3C Document Object Model (DOM) Level 3 Core Specification, W3C Recommendation 07 April 2004

www .w3.org/TR/2004/REC-DOM-Level-3-Core-20040407/

6. Megginson Technologies Ltd SAX: The Simple API for XML www . megginson. com/SAX

7. Hunter J. and McLaughlin B The JDOM Project www.jdom. org

8. Fondazione Ugo Bordoni Torquemada, Web for all www.webxtutti.it/testa_en.htm

9. Watchfire Bobby Accessibility tool bobby.watchfire.com/bobby/html/en/index.jsp

10. CEAPAT, Fundación CTIC, Spanish Ministry of Employment and Social Affairs (IMSERSO) Online Web accessibility test www. tawdis.net

11. Jean Vanderdonckt, Abdo Beirekdar, Monique Noirhomme-Fraiture Automated Evaluation of Web Usability and Accessibility by Guideline Review

Proc. of 4th International Conference on Web Engineering (ICWE 2004), Munich, July 2004, N. Koch, P. Fraternali, M. Wirsing (Eds.), Lecture Notes in Computer Science, Springer-Verlag

12. C. Nentwich, L. Capra, W. Emmerich, and A. Finkelstein xlinkit: A Consistency Checking and Smart Link Generation Service

ACM Transactions on Internet Technology, 2(2):151-185, 2002.

13. Vicente Luque Centeno, Carlos Delgado Kloos, Martin Gaedke, Martin Nussbaumer WCAG Formalization with W3C Standards

WWW2005 conference, May 2005, Chiba, Japan, accepted poster 